\title{
BALANCEDNESS AND THE LEAST LAPLACIAN EIGENVALUE OF SOME COMPLEX UNIT GAIN GRAPHS
}

\author{
Francesco BeLARdo \\ Maurizio Brunetti \\ Department of Mathematics and Applications \\ University 'Federico II', Naples, Italy \\ e-mail: fbelardo@unina.it, mbrunett@unina.it \\ AND \\ NATHAN REFF \\ Department of Mathematics \\ The College at Brockport: State University of New York \\ Brockport, NY 14420, USA \\ e-mail: nreff@brockport.edu
}

\begin{abstract}
Let $\mathbb{T}_{4}=\{ \pm 1, \pm i\}$ be the subgroup of 4 -th roots of unity inside $\mathbb{T}$, the multiplicative group of complex units. A complex unit gain graph $\Phi$ is a simple graph $\Gamma=\left(V(\Gamma)=\left\{v_{1}, \ldots, v_{n}\right\}, E(\Gamma)\right)$ equipped with a map $\varphi$ : $\vec{E}(\Gamma) \rightarrow \mathbb{T}$ defined on the set of oriented edges such that $\varphi\left(v_{i} v_{j}\right)=\varphi\left(v_{j} v_{i}\right)^{-1}$. The gain graph $\Phi$ is said to be balanced if for every cycle $C=v_{i_{1}} v_{i_{2}} \cdots v_{i_{k}} v_{i_{1}}$ we have $\varphi\left(v_{i_{1}} v_{i_{2}}\right) \varphi\left(v_{i_{2}} v_{i_{3}}\right) \cdots \varphi\left(v_{i_{k}} v_{i_{1}}\right)=1$.

It is known that $\Phi$ is balanced if and only if the least Laplacian eigenvalue $\lambda_{n}(\Phi)$ is 0 . Here we show that, if $\Phi$ is unbalanced and $\varphi(\Phi) \subseteq \mathbb{T}_{4}$, the eigenvalue $\lambda_{n}(\Phi)$ measures how far is $\Phi$ from being balanced. More precisely, let $\nu(\Phi)$ (respectively, $\epsilon(\Phi)$ ) be the number of vertices (respectively, edges) to cancel in order to get a balanced gain subgraph. We show that

$$
\lambda_{n}(\Phi) \leq \nu(\Phi) \leq \epsilon(\Phi) .
$$

We also analyze the case when $\lambda_{n}(\Phi)=\nu(\Phi)$. In fact, we identify the structural conditions on $\Phi$ that lead to such equality.

Keywords: gain graph, Laplacian eigenvalues, balanced graph, algebraic frustration.
\end{abstract}

2010 Mathematics Subject Classification: 05C50, 05C22. 


\section{REFERENCES}

[1] R.B. Bapat, D. Kalita and S. Pati, On weighted directed graphs, Linear Algebra Appl. 436 (2012) 99-111.

doi:10.1016/j.laa.2011.06.035

[2] F. Belardo, Balancedness and the least eigenvalue of Laplacian of signed graphs, Linear Algebra Appl. 446 (2014) 133-147.

doi:10.1016/j.laa.2014.01.001

[3] S. Fallat and Y.-Z. Fan, Bipartiteness and the least eigenvalue of signless Laplacian of graphs, Linear Algebra Appl. 436 (2012) 3254-3267.

doi:10.1016/j.laa.2011.11.015

[4] K. Guo and B. Mohar, Hermitian adjacency matrix of digraphs and mixed graphs, J. Graph Theory 85 (2017) 217-248.

doi:10.1002/jgt.22057

[5] R.A. Horn and C. R. Johnson, Matrix Analysis (Cambridge Univ. Press, New York, 2012). doi:10.1017/CBO9781139020411

[6] D. Kalita, Properties of first eigenvectors and first eigenvalues of nonsingular weighted directed graphs, Electron. J. Linear Algebra 30 (2015) 227-242.

doi:10.13001/1081-3810.3029

[7] N. Reff, Spectral properties of complex unit gain graphs, Linear Algebra Appl. 436 (2012) 3165-3176. doi:10.1016/j.laa.2011.10.021

[8] N. Reff, Oriented gain graphs, line graphs and eigenvalues, Linear Algebra Appl. 506 (2016) 316-328.

doi:10.1016/j.laa.2016.05.040

[9] Y.-Y. Tan and Y.Z. Fan, On edge singularity and eigenvectors of mixed graphs, Acta Math. Sin. (Engl. Ser.) 24 (2008) 139-146.

doi:10.1007/s10114-007-1000-2

[10] Y. Wang, S.-C. Gong and Y.-Z. Fan, On the determinant of the Laplacian matrix of a complex unit gain graph, Discrete Math. 341 (2018) 81-86. doi:10.1016/j.disc.2017.07.003

[11] T. Zaslavsky, Biased graphs. I. Bias, balance, and gains, J. Combin. Theory Ser. B 47 (1989) 32-52. doi:10.1016/0095-8956(89)90063-4

[12] T. Zaslavsky, A mathematical bibliography of signed and gain graphs and allied areas, Electron. J. Combin. (1998) \#DS8.

Received 11 June 2019

Revised 15 October 2019 Accepted 24 October 2019 